\title{
ORIGINAL ARTICLE \\ Correlation of diffusion tensor imaging and phase-contrast MR with clinical parameters of cervical spinal cord injuries
}

\author{
S-Y Kim ${ }^{1,2}$, MJ Shin ${ }^{1}$, JH Chang ${ }^{1}$, C-H Lee ${ }^{1,2}$, Y-I Shin ${ }^{1,2}$, YB Shin ${ }^{1}$ and H-Y Ko ${ }^{1,2}$
}

Study design: This is a cross-sectional study.

Objectives: The goal of this study was to characterize the diffusion properties across segments of the spinal cord and peak cerebrospinal fluid (CSF) velocities in the stenotic spinal canal, and to determine the correlation between these properties and clinical and electrophysiological parameters in patients with cervical spinal cord injury (SCI).

Setting: This study was conducted in the University teaching hospital.

Methods: The study involved 17 patients with cervical SCI. The apparent diffusion coefficient (ADC) and fractional anisotropy (FA) of the spinal cord and peak systolic and diastolic velocities of CSF were measured at the level of maximum compression (region 1) and at the levels above (region 2) and below (region 3) the level of injury with no signal change in conventional magnetic resonance imaging. Neurological and electrophysiological parameters were measured, including American Spinal Injury Association (ASIA) Impairment Scale (AIS), ASIA motor score, ASIA sensory score, Modified Barthel Index, Spinal Cord Independence Measure III (SCIM III), somatosensory evoked potentials (SSEP) and motor evoked potentials (MEP).

Results: The ADC was significantly higher and the FA was significantly lower in regions 1, 2 and 3 of the $\mathrm{SCl}$ patients than in the normal controls ( $P<0.05$ each). FA of the level below correlated with AIS, ASIA sensory score and SCIM III score, and FA of the level above correlated with SSEP latencies and MEP amplitudes $(P<0.05$ each). The reductions in FA correlated with CSF flow, functional measurements and evoked potentials.

Conclusions: Diffusion tensor imaging can be used to quantify the proximal and distal extents of spinal cord damage. Reductions in FA were correlated with CSF flow, functional measurements and evoked potentials.

Spinal Cord (2015) 53, 608-614; doi:10.1038/sc.2015.57; published online 14 April 2015

\section{INTRODUCTION}

Owing to its high soft-tissue resolution, conventional magnetic resonance imaging (MRI) is used routinely in the diagnosis of spinal cord injury (SCI). However, conventional MRI has low sensitivity for diffusion abnormalities in the white matter, limiting the associations between MRI results and clinical status. ${ }^{1,2}$ Diffusion tensor imaging (DTI) with fiber tractography provides a threedimensional model of water diffusion for longitudinal evaluations of the central nervous system. ${ }^{3}$ Although the application of DTI to the human spinal cord is challenging technically, owing to the small cross-sectional area of the spinal cord, cardiac and respiratory motion, and widely varying magnetic susceptibility, ${ }^{4}$ recent developments in magnetic resonance (MR) pulse sequence design have greatly reduced these problems. Moreover, phase-contrast MRI (PC-MRI) is a noninvasive technique that can be used to quantify variations in the flow of the cerebrospinal fluid (CSF) gated with the cardiac cycle. ${ }^{5}$ CSF flow can be influenced by stenotic lesions in the brain and spinal cord. ${ }^{6,7}$

Initially, SCI results in the physical disruption of structures in the spinal cord (primary insult), and it might also induce secondary events that injure intact, neighboring tissues in the epidural, subdural, subarachnoid and intramedullary spaces. ${ }^{8,9}$ Therefore, CSF flow is influenced not only by direct injury to the subarachnoid space (SAS) but also by later secondary events.

Current theories of syrinx formation have hypothesized that a phase offset between the arterial and CSF pulse could be a driving force to encourage movement of CSF into the myelon via the perivascular spaces as the Virchow-Robin space. ${ }^{10}$ Spinal canal stenosis and compression of the spinal cord can alter the velocity and turbulence of the CSF flow, allowing the CSF to penetrate the spinal cord and form intramedullary microcysts, thereby changing its diffusion properties.

Few studies have attempted to correlate DTI parameters with the clinical findings in patients with SCI. ${ }^{11,12}$ Moreover, to the best of our knowledge, no study has examined the correlations of DTI parameters and peak CSF velocities with clinical findings in these patients. Therefore, this study characterized the diffusion properties across the spinal cord and CSF velocities in the stenotic cervical spinal canal of SCI patients, to compare these findings with those in normal subjects, and to determine the correlations between DTI results and clinical parameters, including neurological and electrophysiological findings.

${ }^{1}$ Department of Rehabilitation Medicine, Pusan National University School of Medicine, Yangsan, Korea and ${ }^{2}$ Research Institute for Convergence of Biomedical Science and Technology, Pusan National University Yangsan Hospital, Yangsan, Korea

Correspondence: Dr H-Y Ko, Department of Rehabilitation Medicine, Pusan National University School of Medicine, Geum0-ro, Mulgeum, Yangsan, Gyeongnam 626-770, Korea. E-mail: drkohy@gmail.com

Received 7 July 2014; revised 17 February 2015; accepted 2 March 2015; published online 14 April 2015 
Table 1 Characteristics of patients with spinal cord injury at the time of diffusion tensor imaging and cerebrospinal fluid flow study

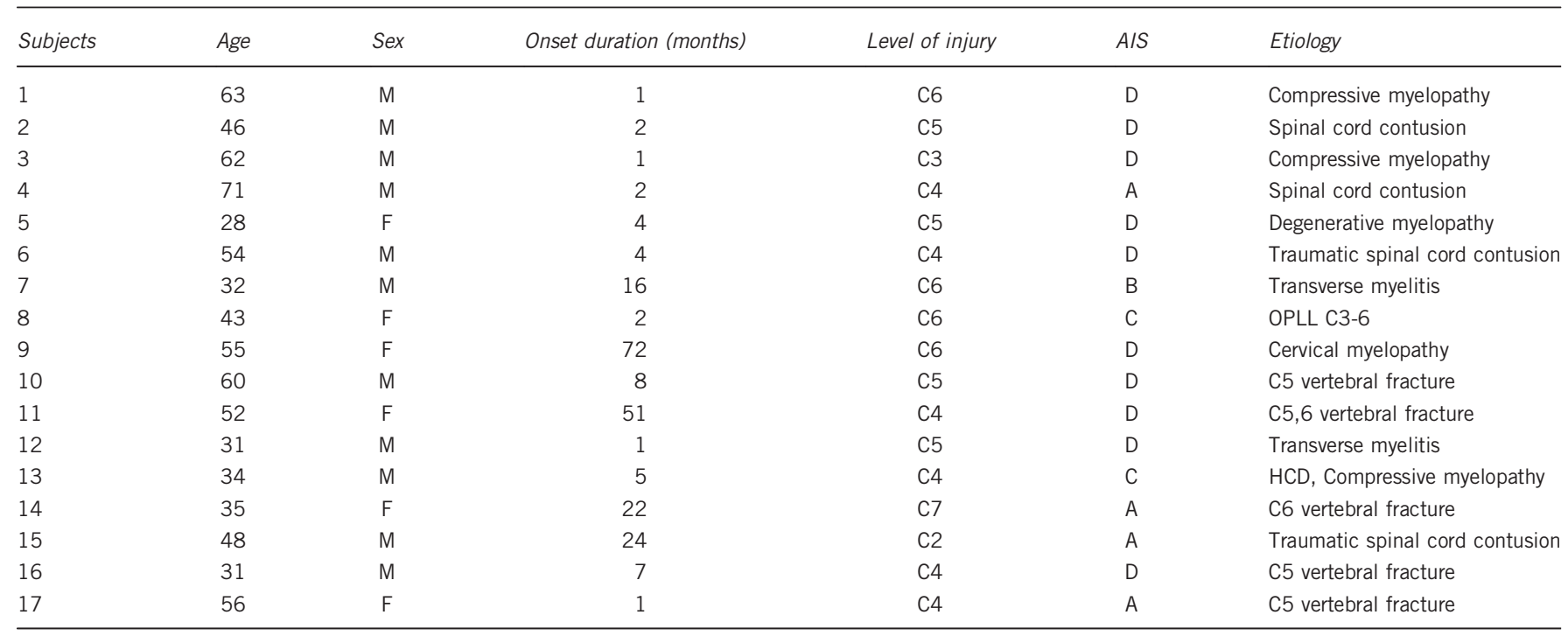

Abbreviations: AIS, ASIA Impairment Scale; F, female; HCD, herniated cervical disc; M, male; OPLL, ossification of posterior longitudinal ligament.

Table $2 \mathrm{FA}$ and $\mathrm{ADC}$ in normal controls and $\mathrm{SCl}$ patients

\begin{tabular}{|c|c|c|c|c|c|c|}
\hline \multirow[t]{2}{*}{ ROI } & \multicolumn{3}{|c|}{ Cervical level of controls } & \multicolumn{3}{|c|}{ Cervical level of SCI patients } \\
\hline & $C 2-3$ & C4-5 & C6-7 & Above & Injury & Below \\
\hline Lateral & $0.72 \pm 0.06$ & $0.69 \pm 0.07$ & $0.71 \pm 0.06$ & $0.54 \pm 0.79$ & $0.57 \pm 0.11$ & $0.58 \pm 0.11$ \\
\hline Ventral & $0.70 \pm 0.07$ & $0.68 \pm 0.08$ & $0.70 \pm 0.06$ & $0.58 \pm 0.01$ & $0.55 \pm 0.61$ & $0.50 \pm 0.08$ \\
\hline Dorsal & $0.71 \pm 0.07$ & $0.74 \pm 0.07$ & $0.73 \pm 0.07$ & $0.60 \pm 0.08$ & $0.61 \pm 0.07$ & $0.54 \pm 0.11$ \\
\hline Lateral & $2.08 \pm 0.26$ & $2.11 \pm 0.22$ & $2.11 \pm 0.31$ & $0.25 \pm 0.43$ & $2.41 \pm 0.50$ & $2.67 \pm 0.57$ \\
\hline Ventral & $2.22 \pm 0.28$ & $2.18 \pm 0.27$ & $2.19 \pm 0.32$ & $2.34 \pm 0.32$ & $2.43 \pm 0.68$ & $2.56 \pm 0.59$ \\
\hline Dorsal & $2.37 \pm 0.32$ & $2.19 \pm 0.26$ & $2.21 \pm 0.25$ & $2.25 \pm .043$ & $2.76 \pm 0.53$ & $2.17 \pm 0.52$ \\
\hline
\end{tabular}

Abbreviations: ADC, apparent diffusion coefficient; FA, fractional anisotropy; ROI, region of interest; $\mathrm{SCl}$, spinal cord injury.

Values are mean \pm s.d.

\section{MATERIALS AND METHODS}

\section{Subjects}

This study enrolled 17 patients ( 11 male, 6 female; mean age, $47.0 \pm 13.4$ years; mean time since injury, $13.1 \pm 19.9$ months) with cervical SCI at levels C2-C7, and 21 age- and sex-matched healthy controls (13 male, 8 female; mean age, $38.5 \pm 15.7$ years). The control subjects had no history of neurological illness and no visible cervical spinal cord lesions on conventional MRI. Patients were excluded if they had undergone vertebral fixation with a metal implant; had complete disruption of the spinal cord or any additional trauma to the peripheral nerves, brain or bone; or were younger than 18 years. Table 1 summarized the profiles and characteristics of the patient subjects.

\section{MRI of DTI}

DTI data for the spinal cord and peak CSF velocities of the spinal canal were obtained using aVerio A Tim system 3-Tesla MR scanner (Siemens, Erlangen, Germany) with a 12-channel head coil and a 4-channel neck coil. DTI of the cervical spinal cord was performed using echo-planar imaging (EPI) and multiecho data image combination (MEDIC) sequences. Intramedullary fractional anisotropy (FA) and apparent diffusion coefficient (ADC) of crosssections of the spinal cord were measured using the MedInria (Inria Sophia Antipolis, Valbonne, France) and FSL (FMRIB, Oxford, UK) software packages. Conventional sagittal T2-weighted images were acquired using a T2-weighted fast spin-echo (FSE) sequence. The parameters used for axial images of DTI sequences were as follows: TR (repetition time) $=5100 \mathrm{~ms}$; TE (echo time) $=77$ $\mathrm{ms}$; NEX (number of average) $=10 ; \quad b$-value $=0,750 \mathrm{~s} \mathrm{~mm}^{-2}$; diffusion direction $=6$; image matrix $=140 \times 36$; FOV (field of view) $=140 \times 36 \mathrm{~mm}^{2}$; slice thickness $=5 \mathrm{~mm}$; total number of slices $=17$; voxel resolution $=$ $1.0 \times 1.0 \times 5 \mathrm{~mm}^{3}$; bandwidth $=916 \mathrm{~Hz}$ per pixel; and TA (total acquisition time) $=6 \mathrm{~min}$ and $2 \mathrm{~s}$. Parameters of the MEDIC (Multiple Echo Data Image Combination) sequence for detailed anatomical images included the following: $\mathrm{TR}=400 \mathrm{~ms} ; \mathrm{TE}=16 \mathrm{~ms}$; flip angle $=30^{\circ} ; \mathrm{NEX}=2$; image matrix $=256 \times 64$; FOV $=140 \times 35 \mathrm{~mm}^{2}$; slice thickness $=5 \mathrm{~mm}$; total number of slices $=17$; voxel resolution $=0.55 \times 0.55 \times 5 \mathrm{~mm}^{3}$; bandwidth $=271 \mathrm{~Hz}$ per pixel; and $\mathrm{TA}=3$ min and $1 \mathrm{~s}$.

$\mathrm{FA}$ and $\mathrm{ADC}$ of the spinal cord in control subjects were measured at spinal levels $\mathrm{C} 2-\mathrm{C} 3, \mathrm{C} 4-\mathrm{C} 5$ and $\mathrm{C} 6-\mathrm{C} 7$. In the patients with SCI, FA and ADC were measured at the level of the spinal cord injury (region 1), and at the levels more than one vertebral segment above (cranial, region 2) and below (caudal, region 3) the level of injury (Table 2), with no signal change on conventional MRI (Figure1).

\section{DTI image analysis}

DTI images were analyzed by an investigator blinded to the clinical and electrophysiological status of each subject. The signal-to-noise ratio was calculated and used as a threshold. Images with signal-to-noise ratio $<20$ were excluded, as were those in which gray and white matter of the spinal cord 


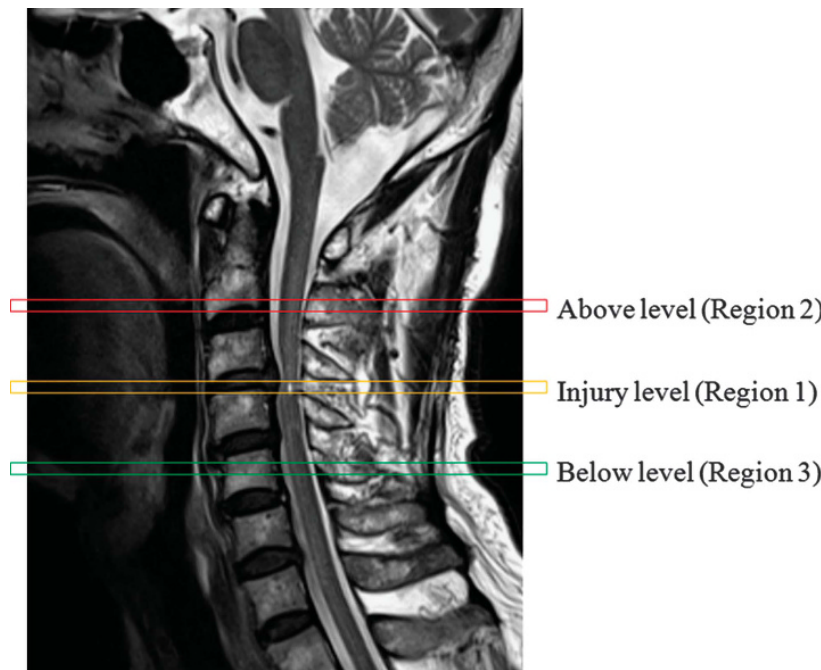

Figure 1 Level of MR DTI and phase-contrast MRI in cervical SCI patients. The three levels of MRI are as follows: region 1, spinal cord injury level or maximum compression level; region 2, more than one vertebral segment above (cranial) the level of spinal cord injury, with no signal change in conventional MRI; region 3, more than one vertebral segment below (caudal) the level of spinal cord injury, with no signal change in conventional MRI.

were indistinguishable because of poor image quality. The sagittal T2-weighted images were screened for the presence of signal abnormalities in the spinal canal, and transverse T2 $(b=0)$ maps in the DTI data sets were screened for lesions. ADC and FA maps were calculated using standard MedInria DTI software within the scanner system. FA and ADC were measured in several regions of interest (ROIs) in cross-sectional areas of the spinal cord. Typical ROIs covering the dorsal (including fasciculus cuneatus and gracilis), ventral (including anterior corticospinal tract) and both lateral areas (lateral corticospinal tract) of the white matter are shown in Figure 2. In the normal control group, ROIs were also placed on gray matter.

\section{PC-MRI}

The patients and normal subjects underwent imaging with the same $3 \mathrm{~T}$ imaging unit. Cardiac gated phase-contrast images of the cervical spinal canal were acquired after the subject had achieved a steady heart rate. CSF flow was analyzed using a fast low-angle shot imaging pulse sequence, with the following parameters: in-plane $\left(\mathrm{TR}=25.85 \mathrm{~ms} ; \mathrm{TE}=8.2 \mathrm{~ms}\right.$; flip angle $=15^{\circ} ; \mathrm{NEX}=1$; image matrix $=205 \times 256$; FOV $=270 \times 270 \mathrm{~mm}^{2}$; slice thickness $=5 \mathrm{~mm}$; total number of slices $=1$; voxel resolution of $1.3 \times 1.1 \times 5 \mathrm{~mm}^{3}$; VENC $=8 \mathrm{~cm} \mathrm{~s}^{-1}$; bandwidth $=195 \mathrm{~Hz}$ per pixel; $\mathrm{TA}=3 \mathrm{~min}$ and $33 \mathrm{~s}$ ) and through-plane $\left(\mathrm{TR}=28.7 \mathrm{~ms} ; \mathrm{TE}=8 \mathrm{~ms} ;\right.$ flip angle $=15^{\circ} ; \mathrm{NEX}=1 ;$ image matrix $=192 \times 256 ;$ FOV $=135 \times 135 \mathrm{~mm}^{2}$; slice thickness $=5 \mathrm{~mm}$; total number of slices $=1$; voxel resolution, $0.7 \times 0.5 \times 5 \mathrm{~mm}^{3}$; VENC $=10,15,20 \mathrm{~cm} \mathrm{~s}^{-1}$; bandwidth $=130 \mathrm{~Hz}$ per pixel; $\mathrm{TA}=4 \mathrm{~min}$ and $48 \mathrm{~s}$ ).

Velocity encoding in the normal control group was obtained at the C2-C3, C4-C5 and C6-C7 spinal levels. In the patients with SCI, CSF velocities were measured at the level of the spinal cord injury (region 1), and at more than one vertebral segment above (cranial, region 2) and below (caudal, region 3) the level of injury, with no signal change on conventional MRI.

Twenty-five image frames were obtained regularly throughout the $\mathrm{R}-\mathrm{R}$ interval, with the $\mathrm{R}$ wave from a peripheral pulse gating.

\section{CSF velocity calculation}

The signal intensity of the CSF anterior to the cord was similar but not always identical to that posterior to the cord throughout the cardiac cycle. ROIs were placed in the anterior SAS of the cervical spine (Figure 2). The ROI statistics output from MedInria included the mean signal intensity of each ROI from each image of the sequence. The positive values on the waveform corresponded a

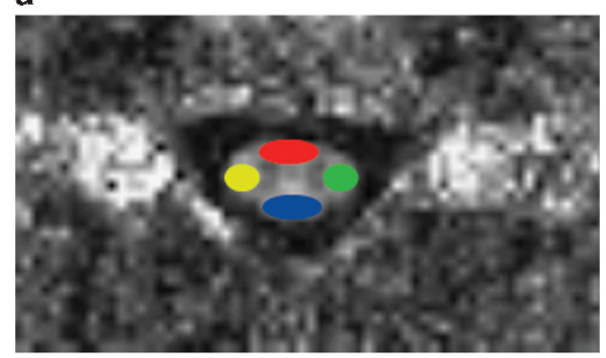

b

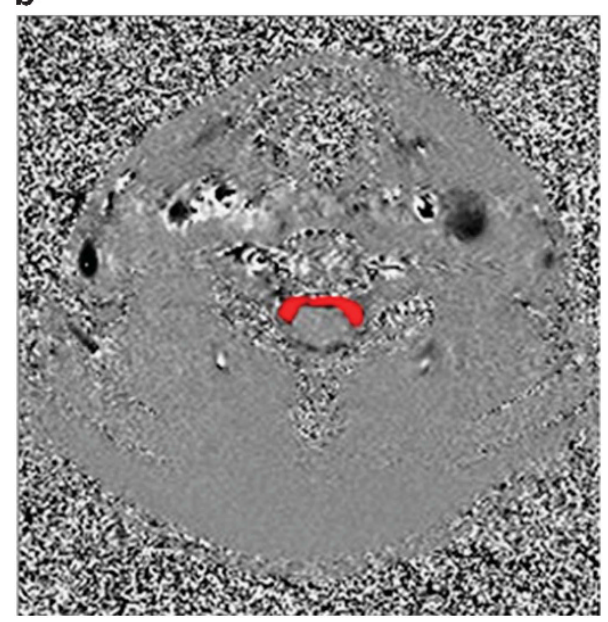

Figure 2 Regions of interest of DTI and phase-contrast MR CSF flow study. (a) ROls of DTI at the cervical spinal cord are shown in red (ventral region), blue (dorsal region), yellow and green (lateral region). (b) ROI of phasecontrast MR CSF flow study placed in the anterior subarachnoid space.

to systolic (craniocaudal) CSF flow and the negative values corresponded to diastolic (caudocranial) CSF flow.

\section{Neurological scoring}

The neurological level of injury was determined using the Standard Neurological Classification of Spinal Cord Injury of the American Spinal Injury Association (ASIA), which was then used to define the lesion site and completeness of injury according to the ASIA Impairment Scale (AIS).

\section{Functional assessment}

Patients with cervical SCI were analyzed using the Modified Barthel Index (MBI) and Spinal Cord Independence Measure III (SCIM III). The MBI is a measure of the activities of daily living, showing the degree of patient independence from assistance.

\section{Electrophysiological measures}

Somatosensory evoked potentials (SSEPs) were recorded by electrical stimulation of the posterior tibial nerve at the medial malleolus, and of the ulnar nerve at the wrist (square wave of $0.1 \mathrm{~ms}$ duration applied at $3 \mathrm{~Hz}$; cathode placed $3 \mathrm{~cm}$ proximal to the anode). The stimulus intensity was adjusted to produce a visible muscle contraction or to a maximum intensity of $40 \mathrm{~mA}$. Scalp electrodes were positioned at $\mathrm{Cz}-\mathrm{Fz}$ to assess the tibial nerve and at $\mathrm{C} 3-\mathrm{C} 4$ to assess the ulnar nerve, as described by the international 10/20 electrode system. Motor evoked potentials (MEPs) for the tibialis anterior muscle and the abductor digiti minimi were recorded after applying single pulse transcranial magnetic stimulation with a double cone coil using a MagPro magnetic stimulator. 


\section{Statistical analysis}

SPSS version 18.0 (SPSS, Chicago, IL, USA) was used for all statistical analyses. The Shapiro-Wilk test was used to test for variable normality. To compare variables between factors, subject, ROI and group at each level, one-way analysis of variance (ANOVA) followed by Scheffe's post hoc test or the Kruskal-Wallis test was used as appropriate. Paired $t$-tests or the MannWhitney $U$ test were used as appropriate to compare differences in CSF flow velocities in the patient and control groups. Spearman's correlation was used to compare the electrophysiological findings and functional measurements with DTI and CSF flow values. Significance was set at $P<0.05$.

\section{RESULTS}

DTI metrics of the control group

Table 2 summarizes DTI parameters according to the cervical vertebral level in the normal control subjects. The mean ( \pm s.d.) FA was $0.72 \pm 0.07$ in the white matter and $0.46 \pm 0.08$ in the gray matter $(P<0.05)$. The mean ADCs were $2.17 \pm 0.26 \times 10^{-3} \mathrm{~mm}^{2} \mathrm{~s}^{-1}$ in the white matter and $2.03 \pm 0.18 \times 10^{-3} \mathrm{~mm}^{2} \mathrm{~s}^{-1}$ in the gray matter $(P<0.05)$. FAs and ADCs of the white matter and the ADCs of the gray matter were similar among the cervical levels in the control subjects $(P>0.05)$. However, FAs in gray matter differed significantly among the cervical levels $(P<0.05)$. FAs and ADCs of white matter were similar for the dorsal, ventral and bilateral ROIs.

a

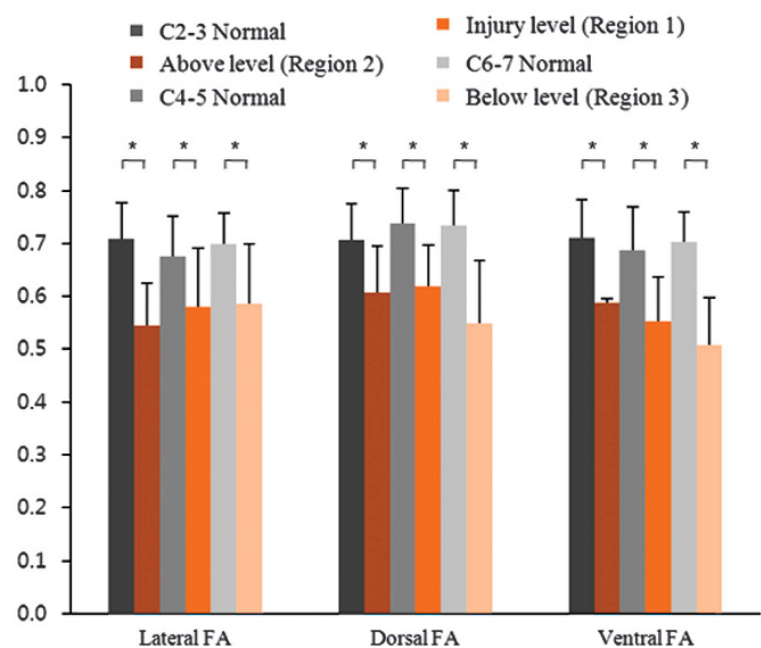

b

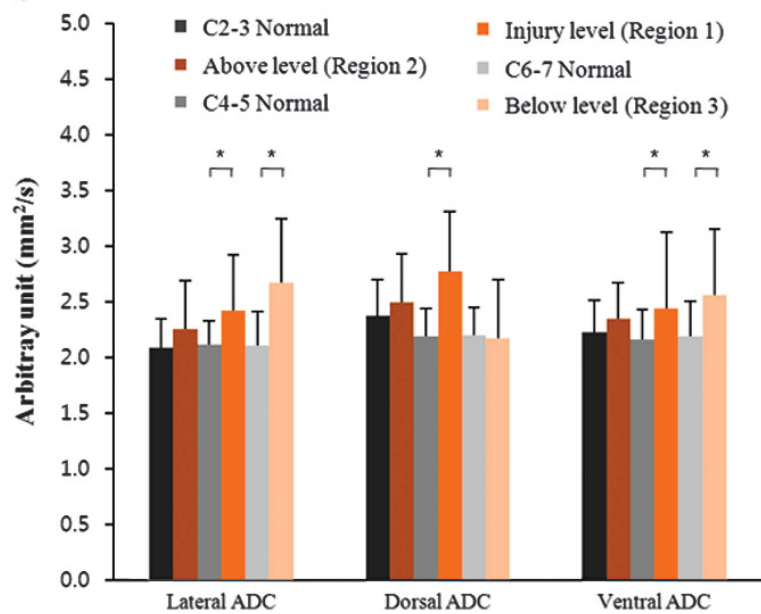

Figure 3 (a) FA and (b) ADC at each spinal cord level of controls and patients $\left({ }^{*} P<0.05\right)$

\section{DTI metrics of the SCI group}

FAs in three regions (above, injury and below levels) were $0.61 \pm 0.09$, $0.65 \pm 0.11$ and $0.59 \pm 0.13$, respectively, and ADCs in these three regions were $2.42 \pm 0.50,2.25 \pm 0.44$ and $2.67 \pm 0.57 \times 10^{-3} \mathrm{~mm}^{2} \mathrm{~s}^{-1}$, respectively. ADCs were significantly higher and FAs were significantly lower in all three regions of SCI patients than in normal controls $(P<0.05$ each). The FAs and ADCs of all ROIs, including those in the lateral, dorsal and ventral areas in all three regions, differed significantly compared with those in the normal control group (Figure 3).

\section{CSF velocity of the control group}

During the cardiac cycle, the signal intensity (velocity) in the CSF anterior to the cord was high (craniocaudal flow) in systolic phase and low (caudocranial flow) in the diastolic phase. The peak systolic and diastolic velocities of the control group, which had mean values of $5.18 \pm 2.02$ and $-7.31 \pm 3.17 \mathrm{~cm} \mathrm{~s}^{-1}$, respectively, are presented in Table 3 . The peak systolic and diastolic velocities did not differ among cervical levels $(P>0.05$ each).

\section{CSF velocity of the SCI group}

The mean values of the peak systolic and diastolic velocities of the patients with SCI were $5.13 \pm 1.48$ and $-6.13 \pm 2.46 \mathrm{~cm} \mathrm{~s}^{-1}$, respectively. At the level of injury (region 1), the mean peak systolic and diastolic velocities were $6.81 \pm 5.02$ and $-8.76 \pm 5.10 \mathrm{~cm} \mathrm{~s}^{-1}$, respectively. In addition, the peak diastolic velocity in below level (region 3) in the SCI group differed from that in the control group $(P<0.05$; Figure 4). Phase-contrast images of all patients revealed a bimodal flow pattern of CSF through the three regions (above, injury and below levels) in the SCI patients and in normal controls. However, images of the patients revealed marked nonuniformity of the signal intensity throughout the three regions during the cardiac cycle (Figure 5).

DTI metrics and CSF velocities of the SCI group

The correlations between DTI parameters and CSF velocities are shown in Table 4. The mean FAs significantly correlated with both the systolic and diastolic velocities at regions 1 and $2(P<0.05$ each). The systolic and diastolic velocities at regions 1 and 3 influenced the changes in FA in regions 1 and $2(P<0.05$ each). ADCs, however, did not significantly correlate with CSF flow velocities.

Table 3 Peak systolic and diastolic velocities of cerebrospinal fluid in the control and patient groups

\begin{tabular}{llll}
\hline & $\begin{array}{c}\text { Peak systolic } \\
\text { velocity }\left(\mathrm{cms}^{-1}\right)\end{array}$ & $\begin{array}{c}\text { Peak diastolic } \\
\text { velocity }\left(\mathrm{cm} \mathrm{s}^{-1}\right)\end{array}$ & ROI area $\left(\mathrm{mm}^{2}\right)$ \\
\hline Control group & & & \\
C2-3 & & & \\
C4-5 & $4.67 \pm 1.78$ & $-6.24 \pm 2.21$ & $55.27 \pm 16.41$ \\
C6-7 & $5.72 \pm 2.35$ & $-8.37 \pm 3.73$ & $44.96 \pm 7.37$ \\
Mean & $5.17 \pm 1.89$ & $-7.36 \pm 3.23$ & $46.72 \pm 10.83$ \\
& $5.18 \pm 2.02$ & $-7.31 \pm 3.17$ & $48.97 \pm 5.52$ \\
Patient group & & & \\
Above level (region 2) & $4.01 \pm 3.18$ & $-5.76 \pm 4.34$ & $48.52 \pm 3.60$ \\
Injury level (region 1) & $6.81 \pm 5.02 *$ & $-8.76 \pm 5.10$ & $37.42 \pm 5.02$ \\
Below level (region 3) & $4.56 \pm 3.02$ & $-3.88 \pm 2.67 *$ & $42.00 \pm 3.51$ \\
Mean & $5.13 \pm 1.48$ & $-6.13 \pm 2.46$ & $42.65 \pm 5.57$ \\
\hline
\end{tabular}

Abbreviation: ROI, region of interest.

Abbreviation: ROI, region
Values are mean \pm s.d.

${ }^{*} P<0.05$. 


\section{Neurologic scoring and functional assessment}

FAs of the ventral area of region 1 significantly correlated with ASIA sensory score $(r=0.687, P=0.009)$. The mean FAs in region 3 significantly correlated with AIS $(r=-0.773, P=0.014)$ and SCIM score $(r=0.680, P=0.044)$. In contrast, FAs and ADCs did not correlate with ASIA motor score or MBI, and CSF peak velocities were not correlated with any of the clinical parameters tested, including AIS, ASIA motor and sensory scores, MBI and SCIM III.

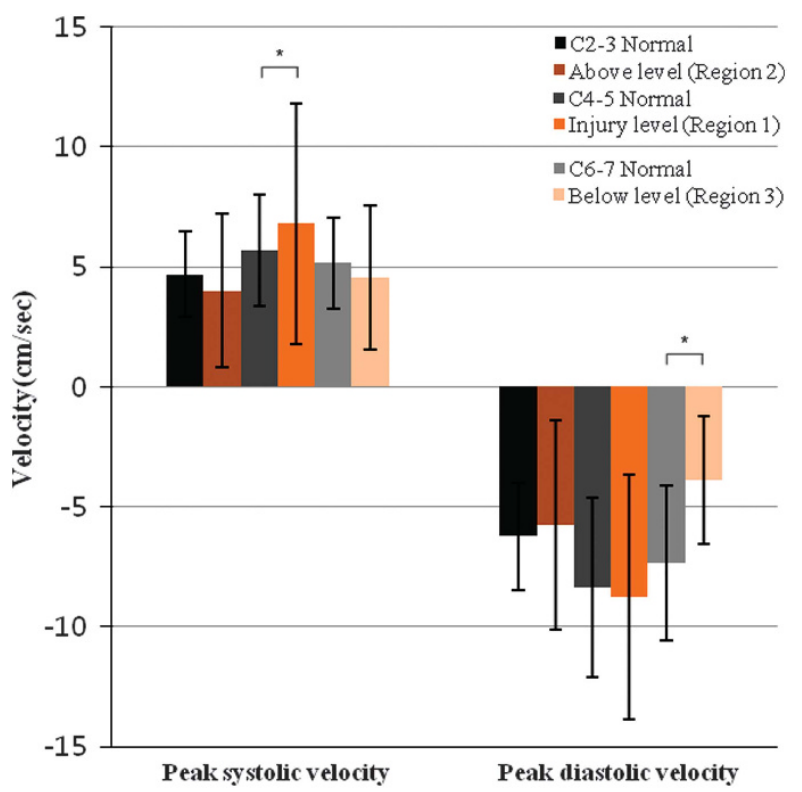

Figure 4 Peak systolic and diastolic velocities of CSF in normal controls and $\mathrm{SCl}$ patients. Significant differences between increased peak systolic velocities at injury level in $\mathrm{SCl}$ patients and significant decreased peak diastolic velocities in $\mathrm{SCl}$ patients at below level $\left({ }^{*} P<0.05\right)$.

\section{Evoked potentials}

The latencies of the ulnar nerve SSEP correlated with FAs in the dorsal area of region $1(r=0.46, P<0.05$; Table 5$)$. In addition, FAs of the lateral and ventral areas of region 1 correlated with tibialis anterior MEPs $(P<0.05)$. CSF velocities in regions 1 and 3 correlated with the amplitudes of tibialis anterior MEPs $(P<0.05)$. In contrast, ADCs did not correlate with SSEP or MEP measurements.

\section{DISCUSSION}

Magnetic resonance determinations of DTI and CSF flow have long been used to evaluate the brain. In contrast, they have not been well established for evaluating the spinal cord, because of the relatively small areas of the spinal cord and SAS, motion artifacts due to respiration and rapid spatial changes in the spinal column. ${ }^{4} \mathrm{FA}$ and ADC are parameters used widely to quantify DTI, with many studies showing changes in these parameters in patients with spinal cord lesions. Decreases in FA have been attributed to the restricted diffusion of water molecules in the compressed cord. Intramedullary edema can destroy the balance between the intracellular and extracellular spaces, with combinations of water molecules and proteins decreasing diffusivity. Because FA values are frequently decreased, while ADC values vary widely, in patients with $\mathrm{SCI}^{13,14} \mathrm{FA}$ values are more sensitive and specific than ADC values for evaluating patients with SCI by MR DTI. ${ }^{15}$

Changes in FA values might indicate cystic necrosis, syrinx or atrophy caused by myelomalacia. ${ }^{16}$ Long-term compression of the spinal cord can cause turbulence in CSF flow, allowing the CSF to penetrate the spinal cord and form intramedullary microcysts not detected by conventional MRI. ${ }^{17}$ PC-MRI determinations of CSF flow have been used to detect Chiari malformations in the cisterna magna and hydrocephalus, which are regions with a relatively wide SAS in the cerebrospinal junction or cerebral aqueduct. ${ }^{5-7}$

The pulsatility of the CSF, initially determined myelographically, is associated with choroid plexus pulsations and is modulated by the respiratory cycle. ${ }^{18,19}$ Cine phase-contrast flow methods can image and perhaps quantify CSF pulsatile flow. ${ }^{5,20}$ To the best of our

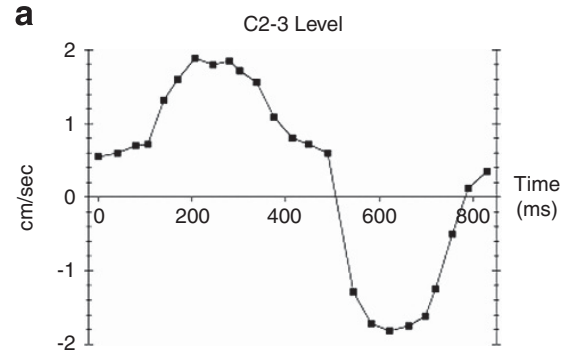

d

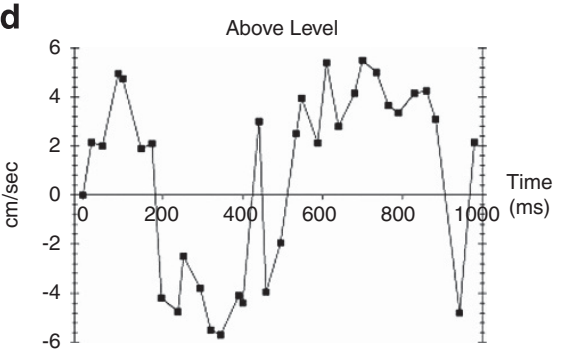

b

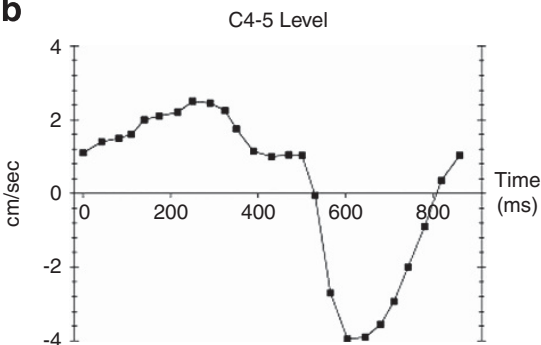

e

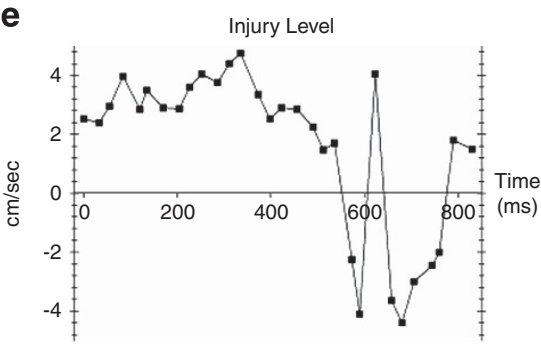

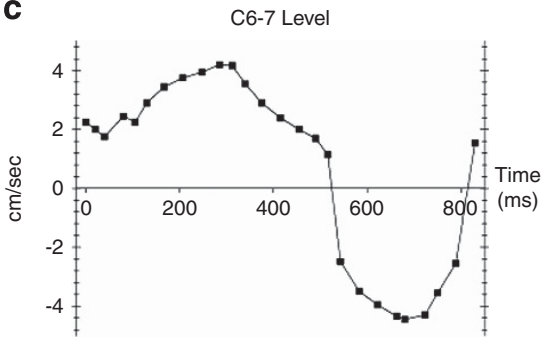

f

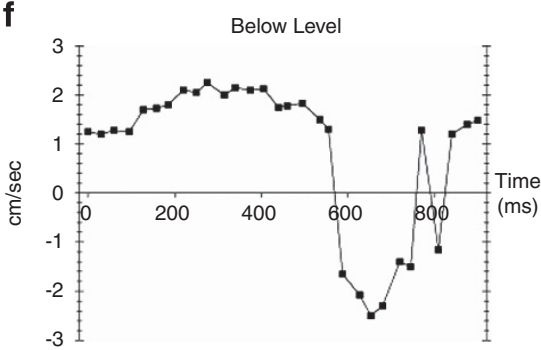

Figure 5 Graphs of CSF velocities in phase-contrast MRI CSF velocities are relatively uniform throughout the subarachnoid space in normal control during cardiac cycle (a-c); CSF velocities are nonuniform in $\mathrm{SCl}$ patient during the cardiac cycle (d-f). 
Table 4 FA and CSF peak velocities in $\mathrm{SCl}$ patients

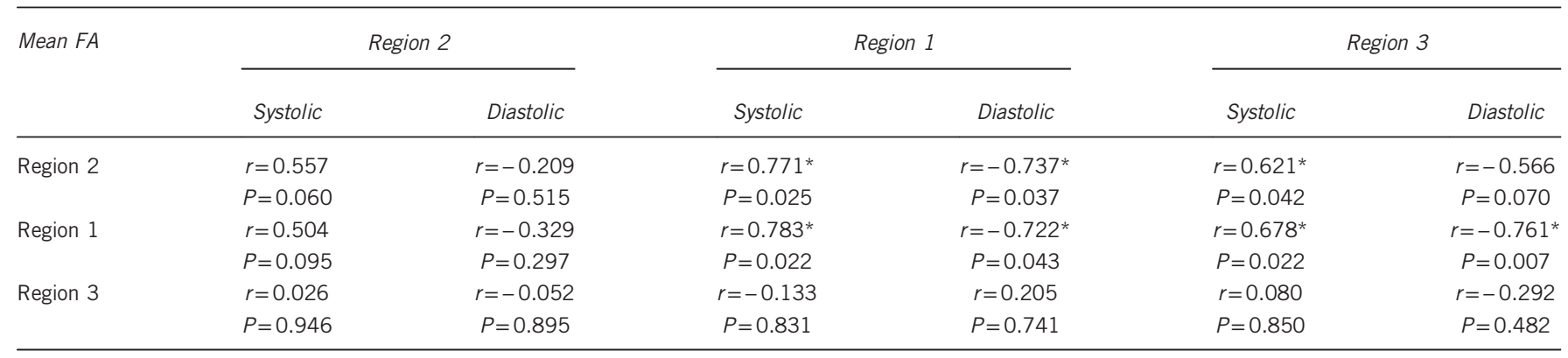

Abbreviations: CSF, cerebrospinal fluid; FA, fractional anisotropy; region 1, injury level; region 2, above level; region 3, below level.

${ }^{*} P<0.05$.

Table 5 Correlation with somatosensory evoked potentials and diffusion properties at spinal cord injury level (region 1 ) in $\mathrm{SCl}$ patients

\begin{tabular}{ccccc}
\hline & Rt. lat. FA & Lt. lat. FA & Dorsal FA & Ventral FA \\
\hline SSEP latency & & & & \\
Rt. ulnar nerve & $r=-0.560^{*}$ & $r=-0.676^{*}$ & $r=-0.641^{*}$ & $r=0.103$ \\
& $P=0.046$ & $P=0.011$ & $P=0.018$ & $P=0.738$ \\
Lt. ulnar nerve & $r=-0.573^{*}$ & $r=-0.675^{*}$ & $r=-0.652^{*}$ & $r=0.073$ \\
& $P=0.041$ & $P=0.011$ & $P=0.016$ & $P=0.814$ \\
Rt. tibial nerve & $r=-0.479$ & $r=-0.500$ & $r=-0.685^{*}$ & $r=0.159$ \\
& $P=0.098$ & $P=0.082$ & $P=0.010$ & $P=0.603$ \\
Lt. tibial nerve & $r=-0.479$ & $r=-0.632^{*}$ & $r=-0.695^{*}$ & $r=0.186$ \\
& $P=0.098$ & $P=0.021$ & $P=0.008$ & $P=0.542$
\end{tabular}

$\begin{array}{ccccc}\text { SSEP amplitude } & & & & \\ \text { Rt. ulnar nerve } & r=0.014 & r=0.135 & r=-0.458 & r=0.322 \\ & P=0.964 & P=0.660 & P=0.116 & P=0.284 \\ \text { Lt. ulnar nerve } & r=-0.262 & r=0.164 & r=-0.468 & r=-0.064 \\ & P=0.387 & P=0.592 & P=0.107 & P=0.834 \\ \text { Rt. tibial nerve } & r=-0.125 & r=-0.151 & r=-0.194 & r=0.358 \\ & P=0.685 & P=0.622 & P=0.526 & P=0.229 \\ \text { Lt. tibial nerve } & r=-0.050 & r=-0.246 & r=-0.279 & r=0.585^{*} \\ & P=0.872 & P=0.417 & P=0.356 & P=0.036\end{array}$

Abbreviations: FA, fractional anisotropy; Lat., lateral; Lt., left; Rt., right; SSEP, somatosensory evoked potentials.

evoked potent
${ }^{*} P<0.05$.

knowledge, no study has assessed the relationships between SCI severity and CSF flow at spinal levels with clinical parameters. Therefore, this study attempted to analyze the relationships between diffusion properties and CSF flow with clinical manifestations.

Quantitative DTI parameters were measured using ROIs at three different anatomic levels of the cervical cord in patients with cervical SCI and healthy controls. The DTI parameters measured in our control group were similar to previous measurements. ${ }^{17}$ The SCI patients had abnormal FAs and ADCs at levels cranial and caudal to the site of injury. In contrast, conventional MRI showed no cord signal abnormalities. These results suggest that FAs and ADCs are sensitive markers of the true extent of cord injury and might also be useful in detecting remote injuries.

Similar to previous findings, $2,11,12,15$ we found that FA was decreased and ADC increased in all three spinal cord regions (that is, the level of injury and levels above and below it). There was a high correlation between SSEP latencies and dorsal FAs in this study. Ulnar SSEP latencies also correlated with lateral FAs, possibly because fibers from cervical levels are located more laterally and were therefore not perfectly matched by the respective dorsal ROI. Assessments of DTI parameters of the lateral and ventral areas, including motor tracts, in region 3 showed that FAs were lower and ADCs higher in this region than in other regions. Wallerian degeneration generally begins to develop in the distal part of an injured neuron just after axonal injury and can be detected at sites distant from the lesion in animal models. ${ }^{21,22}$ Patients with SCI showed greater degrees of disruption and Wallerian degeneration in motor tracts, such as the corticospinal tract, than in sensory tracts at levels caudal to the injured site. ${ }^{23}$

The reported CSF flow velocities in normal control volunteers are between $0.7 \mathrm{~cm} \mathrm{~s}^{-1}$ (diastole) and $1.3 \mathrm{~cm} \mathrm{~s}^{-1}$ (systole) in the midsagittal plane, ${ }^{24}$ and between $2.3 \mathrm{~cm} \mathrm{~s}^{-1}$ (diastole) and $2.1 \mathrm{~cm} \mathrm{~s}^{-1}$ (systole) in the axial plane. ${ }^{20}$ Patients with Chiari I malformations were reported to have peak systole/diastolic CSF velocities in the foramen magnum of $11.7 / 11.6 \mathrm{~cm} \mathrm{~s}^{-1}$, compared with velocities of $4.9 / 5.9 \mathrm{~cm} \mathrm{~s}^{-1}$ in normal controls. ${ }^{24}$ Increased systolic velocities were observed in our SCI patient group at the level of maximum compression, the smallest area of the anterior SAS. However, the diastolic velocities in the lower level were significantly decreased. Although bidirectional CSF flow through the narrow cerebral aqueduct is purely laminar, CSF flow in larger regions, such as the cervical SAS, is often turbulent. ${ }^{18,19}$ This turbulence can cause phase dispersion and signal loss in PC-MRI, resulting in an underestimation of net flow in the cervical SAS. Therefore, the diastolic velocities in below level (region 3) might have been underestimated owing to turbulence through the level of stenosis. Further studies are needed to assess the changes in systolic and diastolic velocities around areas of SCI and spinal stenosis. We found that FAs were correlated with the systolic and diastolic flow velocities at the level of SCI (region 1). SCI can alter FA and CSF flow rate around the level of injury. The mean FA might predict the CSF circulation around areas of SCI.

Several studies have assessed MR DTI and clinical parameters in patients with cervical SCI. For example, lower DTI parameters correlated with the clinical completeness of SCI and with SSEP amplitudes. ${ }^{12}$ ASIA motor scores were correlated with DTI parameters in nonhemorrhagic SCI patients. ${ }^{11}$ Although abnormal cervical levels detected on routine MRI did not correlate with clinical findings and DTI parameters, FAs on DTI correlated with motor function, as did the numbers of imaginary crossing fibers and the rates of connection of fiber tracks in patients with cervical SCI. ${ }^{23}$

This study also evaluated various clinical parameters associated with SCI, including ASIA motor and sensory scores, MBI and SCIM III functional assessments, and evoked potentials. Decreased FAs correlated with AIS, sensory score and SCIM III. In contrast, CSF peak velocities did not significantly correlate with clinical parameters, 
including AIS, ASIA motor and sensory scores, MBI and SCIM III. The correlations of DTI parameters and CSF flow velocities with clinical measurements were weak. Additional studies with more patients are needed to establish these correlations.

Although previous studies ${ }^{11,12,23}$ included patients with metal implants, the MRI signals around the implants might be altered, leading to image deterioration. Such deterioration is more severe in patients with posterior versus anterior implant fixation. This study, however, excluded SCI patients with metal implants including those with anterior and posterior fixation. Compared with previous studies, the exclusion of these patients resulted in a reduction in artifacts resulting from image deterioration. ${ }^{11,12,23}$

Generally, we observed normal values for cervical DTI and CSF flow parameters. DTI parameters, especially FA, can correlate with CSF flow rate and clinical measurements. Alterations in CSF flow on PC-MRI might occur in patients with various types of SCI, including syringomyelia. MRI has shown a pulsatile pattern of the contents of syringomyelic cysts, ${ }^{25}$ which can be detected with T2-weighted spinecho sequence without flow compensation. Finally, measurements of flow turbulence might be useful for assessing patients with pathophysiological abnormalities of the spinal cord.

Important limitations of this study included the small number of patients with heterogeneous types and etiologies of injury, and variations in the time interval between injury and imaging. CSF flow can be influenced by the degree of canal stenosis and etiology (traumatic or nontraumatic). Further prospective studies are needed to determine the relationship between DTI parameters and CSF flow according to etiology and time since injury.

\section{CONCLUSION}

This study found that (1) quantitative DTI analyses are useful in the evaluation of patients with cervical SCI; (2) CSF flow is altered in patients with cervical SCI; and (3) DTI and CSF flow parameters show some correlations with clinical findings such as neurological impairment. More precisely designed studies of patients with acute-stage injuries and including serial follow-up might demonstrate the correlation of these parameters with clinical measurements, delineating the usefulness of DTI and CSF flow studies in patients with cervical SCI.

\section{DATA ARCHIVING}

There were no data to deposit.

\section{CONFLICT OF INTEREST}

The authors declare no conflict of interest.

\section{ACKNOWLEDGEMENTS}

This study was supported by Research Institute for Convergence of Biomedical Science and Technology Grant (30-2014-015), Pusan National University Yangsan Hospital and partly supported for 2 years by Pusan National University Research Grant.
1 Bakshi R, Thompson AJ, Rocca MA, Pelletier D, Dousset V, Barkhof F et al. MRI in multiple sclerosis: current status and future prospects. Lancet Neurol 2008; 7: 615-625.

2 Ellingson BM, Ulmer JL, Kurpad SN, Schmit BD. Diffusion tensor MR imaging in chronic spinal cord injury. AJNR Am J Neuroradiol 2008; 29: 1976-1982.

3 Hagmann P, Jonasson L, Maeder P, Thiran JP, Wedeen VJ, Meuli R. Understanding diffusion MR imaging techniques: from scalar diffusion-weighted imaging to diffusion tensor imaging and beyond. Radiographics 2006; 26: S205-S223.

4 Clark CA, Werring DJ. Diffusion tensor imaging in spinal cord: methods and applications - a review. NMR Biomed 2002; 15: 578-586.

5 Nitz WR, Bradley WG, Watanabe AS, Lee RR, Burgoyne B, O'Sullivan RM et al. Flow dynamics of cerebrospinal fluid: assessment with phase-contrast velocity MR imaging performed with retrospective cardiac gating. Radiology 1992; 183: 395-405.

6 Haughton VM, Korosec FR, Medow JE, Dolar MT, Iskandar BJ. Peak systolic and diastolic CSF velocity in the foramen magnum in adult patients with Chiari malformations and in normal control participants. AJNR Am J Neuroradiol 2003; 24: 169-176.

7 Koc K, Anik Y, Anik I, Cabuk B, Ceylan S. Chiari 1 malformation with syringomyelia: correlation of phase-contrast cine MR imaging and outcome. Turk Neurosurg 2007; 17 183-192.

8 Noble LJ, Wrathall JR. Correlative analyses of lesion development and functional status after graded spinal cord contusive injuries in the rat. Exp Neurol 1989; 103: 34-40

9 Noble LJ, Wrathall JR. Distribution and time course of protein extravasation in the rat spinal cord after contusive injury. Brain Res 1989; 482: 57-66.

10 Mautes AE, Weinzierl MR, Donovan F, Noble LJ. Vascular events after spinal cord injury: contribution to secondary pathogenesis. Phys Ther 2000; 80: 673-687.

11 Cheran S, Shanmuganathan K, Zhuo J, Mirvis SE, Aarabi B, Alexander MT et al. Correlation of MR diffusion tensor imaging parameters with ASIA motor scores in hemorrhagic and nonhemorrhagic acute spinal cord injury. J Neurotrauma 2011; 28: 1881-1892.

12 Petersen JA, Wilm BJ, von Meyenburg J, Schubert M, Seifert B, Najafi Y et al. Chronic cervical spinal cord injury: DTI correlates with clinical and electrophysiological measures. J Neurotrauma 2012; 29: 1556-1566.

13 Ozanne A, Krings T, Facon D, Fillard P, Dumas JL, Alvarez $\mathrm{H}$ et al. MR diffusion tensor imaging and fiber tracking in spinal cord arteriovenous malformations: a preliminary study. AJNR Am J Neuroradiol 2007; 28: 1271-1279.

14 Renoux J, Facon D, Fillard P, Huynh I, Lasjaunias P, Ducreux D. MR diffusion tensor imaging and fiber tracking in inflammatory diseases of the spinal cord. AJNR Am J Neuroradiol 2006; 27: 1947-1951.

15 Facon D, Ozanne A, Fillard P, Lepeintre JF, Tournoux-Facon C, Ducreux D. MR diffusion tensor imaging and fiber tracking in spinal cord compression. AJNR Am J Neuroradiol 2005; 26: 1587-1594.

16 Tsuchiya K, Katase S, Fujikawa A, Hachiya J, Kanazawa H, Yodo K. Diffusion-weighted MRI of the cervical spinal cord using a single-shot fast spin-echo technique: findings in normal subjects and in myelomalacia. Neuroradiology 2003; 45: 90-94.

17 Demir A, Ries M, Moonen CT, Vital JM, Dehais J, Arne P et al. Diffusion-weighted MR imaging with apparent diffusion coefficient and apparent diffusion tensor maps in cervical spondylotic myelopathy. Radiology 2003; 229: 37-43.

18 Du Boulay GH. Pulsatile movements in the CSF pathways. Br J Radiol 1966; 39: 255-262.

19 Gilland O, Chin F, Anderson WB, Nelson JR. A cinemyelographic study of cerebrospinal fluid dynamics. Am J Roentgenol Radium Ther Nucl Med 1969; 106: 369-375.

20 Enzmann DR, Pelc NJ. Normal flow patterns of intracranial and spinal cerebrospinal fluid defined with phase-contrast cine MR imaging. Radiology 1991; 178: 467-474.

21 DeBoy CA, Zhang J, Dike S, Shats I, Jones M, Reich DS et al. High resolution diffusion tensor imaging of axonal damage in focal inflammatory and demyelinating lesions in rat spinal cord. Brain 2007; 130: 2199-2210.

22 Zhang J, Jones M, DeBoy CA, Reich DS, Farrell JA, Hoffman PN et al. Diffusion tensor magnetic resonance imaging of Wallerian degeneration in rat spinal cord after dorsal root axotomy. J Neurosci 2009; 29: 3160-3171.

23 Chang Y, Jung TD, Yoo DS, Hyun JK. Diffusion tensor imaging and fiber tractography of patients with cervical spinal cord injury. J Neurotrauma 2010; 27: 2033-2040.

24 Armonda RA, Citrin CM, Foley KT, Ellenbogen RG. Quantitative cine-mode magnetic resonance imaging of Chiari I malformations: an analysis of cerebrospinal fluid dynamics. Neurosurgery 1994; 35: 214-223. discussion 223-224.

25 Enzmann DR, O'Donohue J, Rubin JB, Shuer L, Cogen P, Silverberg G. CSF pulsation within nonneoplastic spinal cord cysts. AJR Am J Roentgenol 1987; 149: 149-157. 